\title{
Insect Venom Toxin Peptides, its Antimicrobial Effects and Host Immune Responses: A Review
}

\section{Simran Sharma and Ravi Kant Upadhyay*}

Department of Zoology, Deen Dayal Upadhyaya Gorakhpur University, UP, India

*Corresponding Author: Ravi Kant Upadhyay, Department of Zoology, Deen Dayal

Upadhyaya Gorakhpur University, UP, India.
Received: March 01, 2021

Published: March 25, 2021

(C) All rights are reserved by Simran Sharma and Ravi Kant Upadhyay.

\begin{abstract}
Present review article explains antimicrobial activity of hymenopteran insect venoms. Insects mostly bees, wasps, hornets use venom toxins to defend their hive, nests and mainly colonies in territory. These insects sting very swiftly and inflict venom in its prey. Insect venom glands secret multiple toxin components which are powerful weapons secreted from venom glands. Bees, wasps, and hornet inflict venom for self and territorial defense and impose physiological alterations with multiple symptoms in intruders; but envenomation does not transmit any pathogen. Bee venom severely affects cytoskeletal system and impairs nerve cell function that results in organ paralysis and deformity and even death after multiple bites. In bees severity of microbial infection is increased due to impact of biotic and abiotic factors. Few phenolic compounds such as flavonoids mixed in honey, propolis, and royal jelly assist in making antiviral defense. Insects secrete antimicrobial peptides in venom which protect themselves from infection by generating immune response against pathogens. Purified venom toxins exhibit antibacterial, antifungal and antiviral defense. No doubt venom components from honey bees, wasps and hornets can be used as templates for generation of new therapeutic agents and biopesticides for insect pest management. For this purpose venom gland genome is to be sequenced for finding regulatory genes which synthesize diverse venom components. This overall information generated at molecular level could be used for making diagnostic kits to detect effect of bee venom allergens.
\end{abstract}

Keywords: Hymenopteran Insects; Venom; Toxins; Biological and Therapeutic Effects

\section{Abbreviations}

MAC-1: Macropin; AMPs: Antimicrobial Peptide; AcKTSPI: Kazaltype Serine Protease Inhibitor; PNG-1: Panurgines, Polydim-I (Polybia dimorpha); Equmenine mastoparan-EM1 and Equmenine mastoparan-EM2 Peptides; MP-V1: Mastoparan V1; AMG: Anterior Main Gland; PMG: Posterior Main Gland; Polybia-CP: Polybia paulista, Melittin; PLA2: Phospholipase A2; VSV: Vesicular Stomatitis Virus; RSV: Respiratory Syncytial Virus; HSV: Herpes Simplex Virus; JV: Junín Virus; RSV: Respiratory Syncytial Virus; VSV: Vesicular Stomatitis Virus; TMV: Tobacco Mosaic Virus; HIV: Human
Immunodeficiency Virus; CXCR4: Chemokine Receptor; BmNPV: Bombyx Mori Nucleopolyhedrovirus; RFP: Red Fluorescent Protein; ACPs: Anti-Cancer Peptides; Jak/STAT: Janus kinase/signal Transducer and Activator Of Transcription; JNK: c-Jun N-terminal Kinase; MAPK: Mitogen-Activated Protein Kinases; SF-21AE: Spodoptera frugiperda; and Lymantria dispar (IPL-Ldfbc1) Cells

\section{Introduction}

Hymenopteran insects such as honey bees, wasps and hornets are highly venomous. These make hives and protect them. After

Citation: Simran Sharma and Ravi Kant Upadhyay. "Insect Venom Toxin Peptides, its Antimicrobial Effects and Host Immune Responses: A Review". Acta Scientific Microbiology 4.4 (2021): 140-156. 
feeling a little disturbance in surroundings honey bees and wasps attack in groups while hornets attack in group of two or three insects. Envenomation by hornets is highly painful to human than the wasp stings because hornet venom contains acetylcholine $5 \%$ in amount. Various species of honey bees are major plant pollinators and collect nectar from garden flowers and from agriculture fields. Due to rising pollution and depletion of foraging sites, wild bee colonies are decreasing at alarming rate. This local decline is going on at larger scale in some of the geographical regions. For population decline in wild bees many factors like viral diseases and temperature variations and predators are responsible. In has been noted that positive sense strand RNA viruses are targeting bees and infecting them. After bee bite people experience multiple symptoms, but insect envenomation do not transmit the virus. Bee venom severely affect cytoskeletal system and nerve cells that results in organ paralysis and deformity and even death after multiple bites. In case of microbial infection severity is increased due to impact of biotic and abiotic factors. Bees protect themselves from infection by using defensins and generating immune response against pathogens.

This article highlights antiviral defense found in bees, including the Western honey bee (Apis mellifera), the Eastern honey bee (Apis cerana) and bumble bee species (Bees Bombus sp.). Though wild bees maintain antiviral defense but for controlling their heavy decline and mitigation of bee losses immune -genetic studies are to be done to establish relationship between socialization of bees and immune function. Primarily eusocial wasps have an important role in the origin of eusociality that comes after cooperative foraging and social defense. The uniqueness is that all members of a group get infection in maintaining socialization for which they synthesize defensins. These are used to make immune defense. Moreover, human beings use antibiotics to defend themselves in response to viral infection; similarly, bees synthesize defense proteins to encounter the pathogen. Antibacterial peptides synthesized in insects in response to various pathogens were found highly effective and could be used as broad spectrum anti-microbial agents. These also become new alternative of synthetic antibiotics.

Antibacterial peptides from insects can be used as therapeutic agents or potential drugs which could target multidrug resistant pathogens. Today multiple drug resistance in microbes is a biggest challenge for the pharmaceutical companies and researchers.
Based on research outcomes spider venom toxins are also found highly effective against various pathogens and could be used as useful resource for drug development. These were found highly effective against drug-resistant microorganisms. The primary functions of solitary wasp are protection of hive while social wasp protects every member and its progenies by developing defense against predators and pathogens. Solitary wasps use stinging for paralyzing the prey while social wasps defend their colonies having progenies from vertebrate predators. There occurs a significant difference in venom components on the basis of socialization hyalurinidase, phospholipase A2 and metalloendopeptidase are common venom components found in both solitary and social wasp. Venom peptides from wasps show diverse biological activities.

Solitary wasps possess more diverse bioactive components that are used in prey inactivation by physiological manipulation. Solitary wasp venom contains pompilidotoxin and dendrotoxin peptides which are neurotoxic in nature. These also contain insulin peptide binding proteins. Insulin peptide binding proteins is highly specific to solitary wasps Contrary to this, venom allergen 5 proteins; venom acid phosphatases, and various phospholipases, were found relatively more specific to social wasp venom.

\section{Antimicrobial peptides}

Antimicrobial peptides are commonly found in all insect groups, and used in making immune defense against various pathogens. These peptides were found highly active against Gram-negative and Gram-positive bacteria, fungi and viruses, etc. These insect origin AMPs show low toxicity to mammalian cells. Macropin (MAC-1) is an antimicrobial peptide (AMPs) isolated from the venom of the solitary bee Macropis fulvipes. It is a lecuine and lysine rich toxin peptide having 13 amino acids in its structure. MAC-1 shows broad spectrum activity against both Gram negative and Gram positive bacteria. Similarly, a peptide isolated from bee (Apis cerana) venom acts as a Kazal-type serine protease inhibitor (AcKTSPI) and found active against Gram-positive bacteria [3] (Table 1).

Panurgines are antimicrobial peptides (AMPs) isolated from the venom of the wild bee Panurgus calcaratus. These peptides display strong antimicrobial activity against Gram-positive and Gram-negative bacteria and many fungal strains. These show low hemolytic activities against human erythrocytes [4]. PNG-1 is $\alpha$-helical amphipathic dodecapeptide shows amino acid sequences 


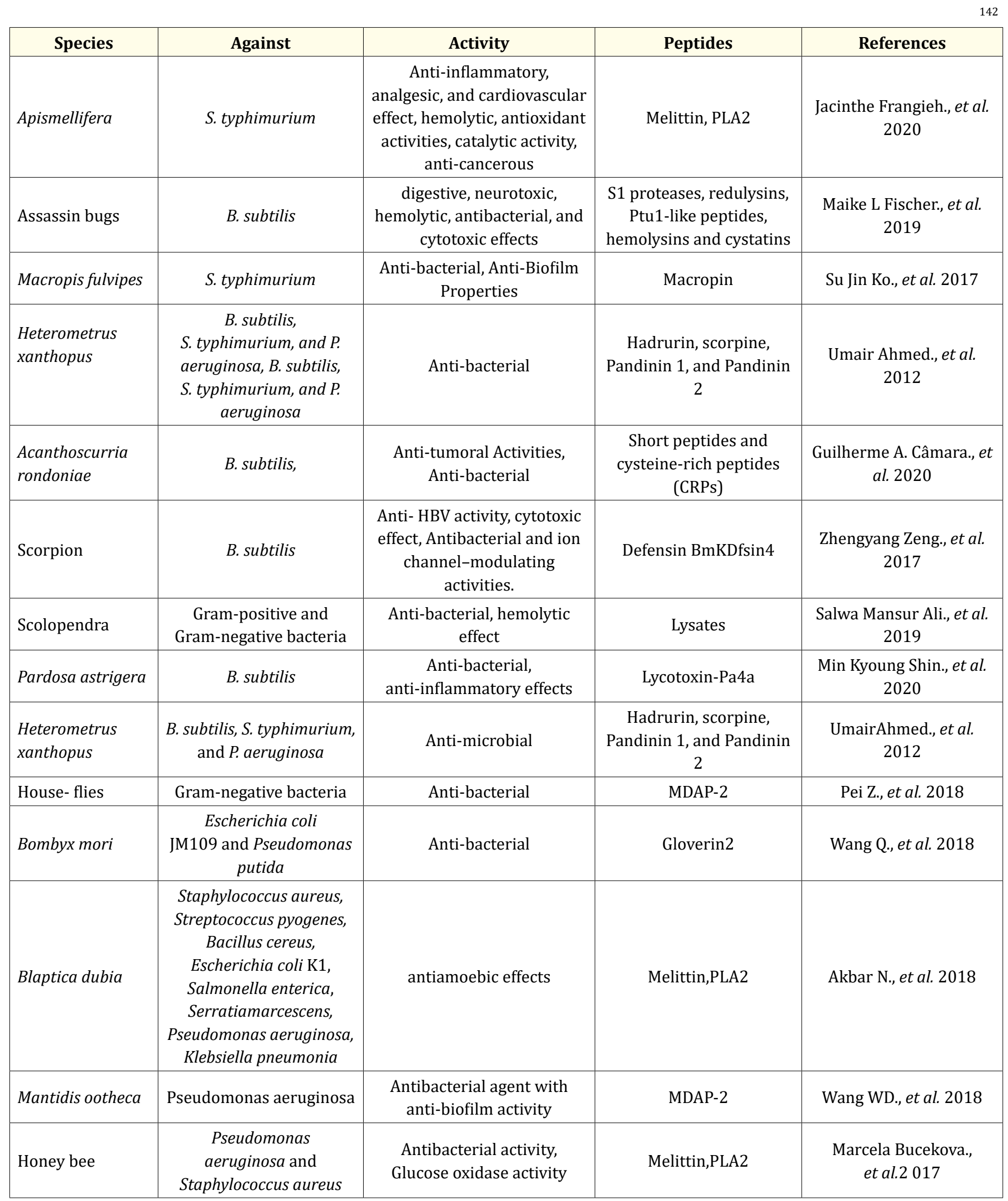




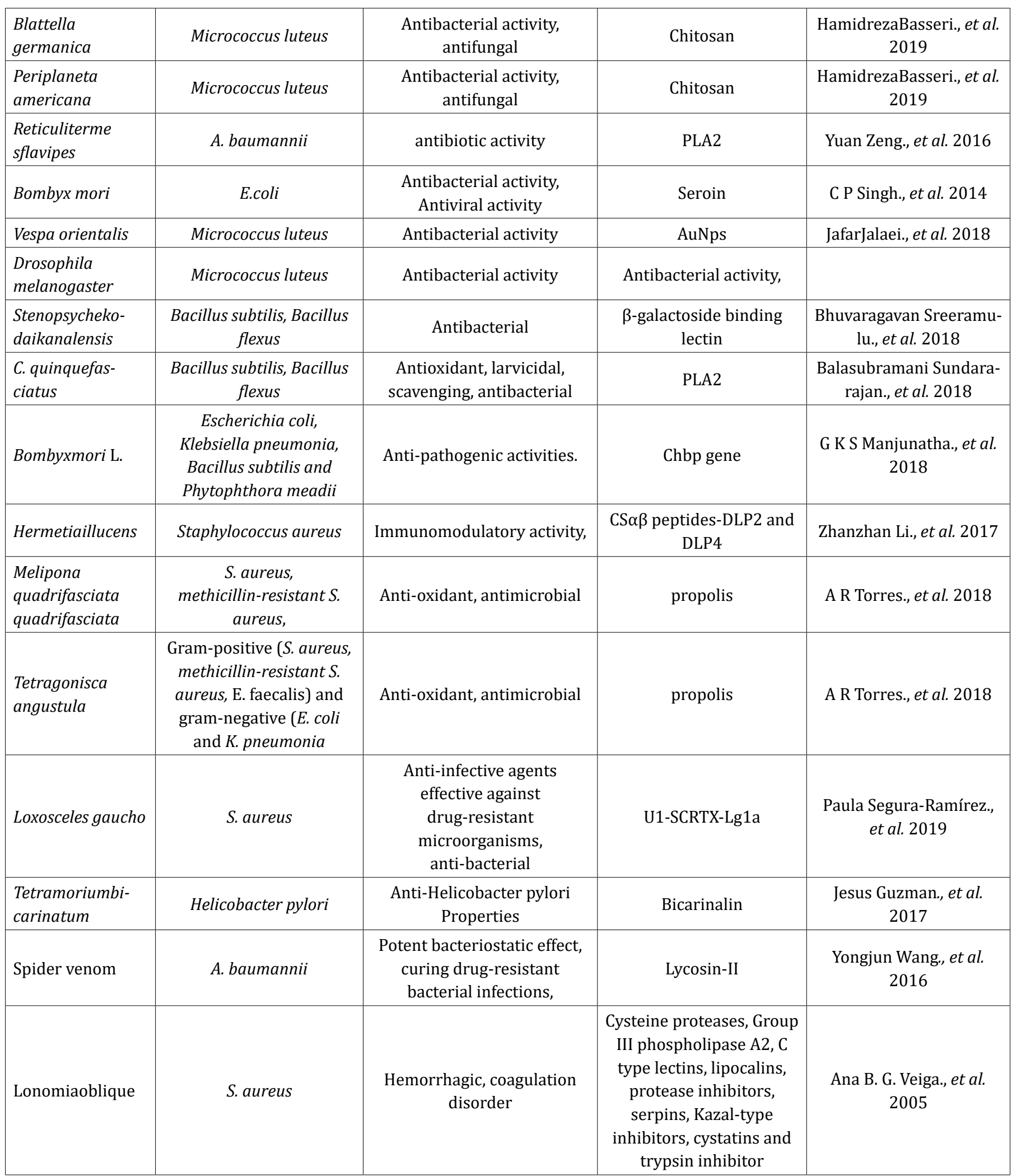




\begin{tabular}{|l|c|c|c|c|}
\hline \multicolumn{1}{|l|}{ Scorpion toxins } & S. aureus & $\begin{array}{c}\text { Neurotoxic, Antibacterial } \\
\text { ancestral activity }\end{array}$ & defensin & $\begin{array}{c}\text { Shangfei Zhang., } \text { et al. } \\
2018\end{array}$ \\
\hline $\begin{array}{l}\text { Latrodectus } \\
\text { geometricus }\end{array}$ & A. baumannii & $\begin{array}{c}\text { Latrotoxins, } \\
\text { Enzymatic activity, toxicity, } \\
\text { and antibacterial activity } \\
\text { apolins, } \\
\text { hemocyanins, chitinases, } \\
\text { arginine kinase, allergen } \\
\text { antigen 5-like protein, } \\
\text { astacin-like }\end{array}$ & $\begin{array}{c}\text { Khamtorn, Pornsawan., } \\
\text { et al. 2020 } \\
\text { metalloproteases, and } \\
\text { serine proteases. }\end{array}$ & Latroeggtoxin-IV \\
\hline $\begin{array}{l}\text { L. tredecimgut- } \\
\text { tatus }\end{array}$ & A. baumannii & Antibacterial & QianLei., et al. 2015 \\
\hline
\end{tabular}

Table 1: Antibacterial venom toxins insects.

LNWGAILKHIIK-NH $\mathrm{N}_{2}$. Lasiocepsin a 27-residue antimicrobial peptide was isolated from Lasioglossum laticeps (wild bee) venom. It also exhibits antibacterial and antifungal activity [5]. Polybia-MPII shows very high antimicrobial potential and shows comparatively low erythrocyte hemolysis than other peptides of this same family [6]. Similar antimicrobial activity is reported in mastoparans isolated from the venom of the social wasp Pseudopolybia vespiceps against Staphylococcus aureus and Mycobacterium abscessus, Candida albicans and Cryptococcus neoformans in vitro. These antimicrobial peptides display pore-forming ability in membrane and could be used as antimicrobial drugs.

Polybia dimorpha is a neotropical wasp, secretes an antimicrobial peptide Polydim-I in venom [7]. This is a cationic peptide displays potent antimicrobial activity against different microorganisms. It disrupts cell wall and but shows no cytotoxic effects in mammalian cells [8]. MP-V1 (Mastoparan V1) is an antimicrobial peptide isolated from the venom of the social wasp Vespula vulgaris. It was found active against Salmonella typhimurium infection [9]. Few $\alpha$-helical peptides also found in Equmenine wasp venom $[9,10]$. Kazal-type serine proteases found in insect venoms exhibit thrombin, elastase, plasmin, proteinase $\mathrm{K}$, or subtilisin A inhibition activity. Both Equmenine mastoparan-EM1 and Equmenine mastoparan-EM2 peptides exhibit potent antibacterial activity [11]. Antimicrobial peptides (AMPs) are also identified in scorpion and spider venoms. These show potent antimicrobial activity against pathogenic bacteria. Scorpion venom contains some bi-functional peptides, which could be used to synthesize/engineer new pro- teins with an ancestral activity [12]. These insect derived short antimicrobial peptides have shown better bactericidal activity against multidrug resistant $M$. tuberculosis than that of the conventional antibiotics ethambutol, isoniazid and rifampicin [12]. Both peptides Pin2 and Pin2 have shown much potent antimicrobial activity and could be used as an alternative of antibiotics to treat tuberculosis [13]. These show very low hemolytic effects [14-17].

Spider Laches anatarabaevi synthesize cytolytic peptides which act synergistic action when used with neurotoxins. Spiders use venom peptides to paralyze prey or deter aggressors. This is a bifunctional peptide which binds to bacterial membrane and found active against Gram-negative and Gram-positive bacteria. Lasiocepsin is isolated from Lasioglossum laticeps (wild bee) venom it contains 27 amino acid residues. This peptide shows antibacterial and anti-fungal activity. Lasiocepsin structurally belongs to the ShK family, it shows strong affinity with anionic phospholipids of membrane [15]. Lasiocepsin peptide permeabilizes through membrane of Gram-negative bacteria and kills them. Lasiocepsin peptide interacts with phospholipids at $\mathrm{N}$ terminus site; its penetration power depends on presence of cardiolipin that found at the poles of bacterial cells [16]. Solitary wasps use sting to paralyze the predator but these do not feed upon it. But spiders paralyze their prey and feed them to their larvae.

Vespula vulgaris secrete MP-V1 antimicrobial peptide that was found active against Salmonella infection [17]. Polydim-I is a cationic peptide shows powerful antimicrobial activity against different microorganisms [18]. Equmenine wasps Anterhynchium 
flavor marginatum micado venom contains antimicrobial $\alpha$-helical peptides as the major peptide component that also showed antimicrobial, histamine-releasing, and hemolytic activities. Wasp venom peptide Mastoparan-EM1 and EM2 display mast cell degranulation activity [19]. EMP-EM peptides strongly bind with bacterial and mammalian cell membranes. The paper wasp Polistes dominulus venom was found effective against polymicrobial disease of vineyards [20].

Wasp venoms are complex mixtures of proteinacious and nonproteinacious substances. Endoparasitoid wasp, Microplitis mediator secretes VRF1, a metalloprotease homolog venom protein. It modulates egg encapsulation in its host, the cotton bollworm, Helico verpa armigera. This endoparasitoid wasp venom protein interferes with the Toll signaling pathway in the host hemocytes [21]. Similarly, another protein Edin express in the fat body of hosts and regulate plasmatocyte numbers. It also regulates mobilization of sessile hemocytes in Drosophila larvae [22]. Insect venom is a rich source of diverse peptides which could be used for drug design and innovative therapeutic discoveries [23].

Bee and wasp venom contains several biologically active biomolecules such as peptides and enzymes. These show strong therapeutic potential against micro-organisms mainly causative agents of infectious diseases. Bee venom components also found active against different cancer cell types [24]. Insect venom toxins could be used to cure of central nervous system diseases i.e. Parkinson's disease, Alzheimer's disease, and amyotrophic lateral sclerosis in human. The venoms from the anterior main gland (AMG) and posterior main gland (PMG) of the reduviid bugs Platymeris biguttatus L. and Psytalla horrida Stål secretes distinct protein mixtures possesses S1 proteases, redulysins, Ptu1-like peptides, and uncharacterized proteins, whereas AMG venom contained hemolysins and cystatins. It shows neurotoxic, hemolytic, antibacterial, and cytotoxic effects [25].

\section{Antibacterial activity}

Bee and wasp venom contains several biologically active biomolecules such as peptides and enzymes. These show strong therapeutic potential against micro-organisms mainly causative agents of infectious diseases. Polybia-CP isolated from Polybia paulista shows strong antibacterial potential against both Gram-positive and Gram-negative bacteria. This is a membrane active peptide and passes through membrane of bacteria. It shows strong action against drug resistant bacteria [26] (Figure 1). The wasp $A$. baumannii MPs Agelaia-MPI and Polybia-MPII had better action against MDR (multidrug-resistant) [27]. Anoplin and its several analogs showed antibacterial activity against methicillin-resistant Staphylococcus aureus ATCC 33591 (MRSA), Escherichia coli (ATCC 25922), Pseudomonas aeruginosa(ATCC 27853), vancomycin-resistant Enterococcus faecium (ATCC 700221) [28]. Similarly, opistoporin 1 and parabutoporin isolated from scorpion Opistophtalmus carinatus inhibit growth of Gram-negative bacteria [29] (Table 1) (Figure 1).

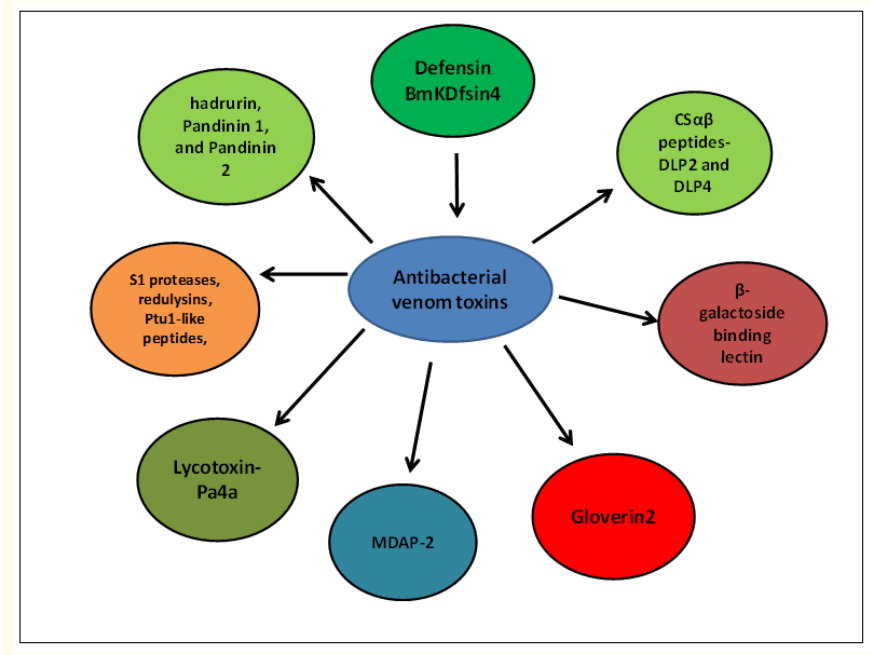

Figure 1: Showing antibacterial activity of insect venom toxins.

\section{Antifungal activity}

A synthetic decapeptide antifungal activity against Penicillium rot of apples [30], $\alpha$-hefutoxin 1 and analogues showed inhibitory effect on the oncogenic channel $\mathrm{K}_{\mathrm{v}} 10.1$ [31]. A peptide analog from the mastoparan wasps (MK58911) showed antifungal activity against Cryptococcus spp. and Paracoccidioides spp. This peptide could be used as a new antifungal drug to treat systemic mycoses [32]. Polybia-CP is an antimicrobial cationic peptide purified from the venom of the social wasp Polybia paulista. It shows potent antifungal activity against Candida albicans (C. albicans) and Candida glabrata (C. glabrata). Polybia-MPI, inhibits growth of $C$. glabrata, and could be used for development of antifungal agents. Polybia- 
CP enhances the production of cellular reactive oxygen species that aid to its anti-fungal activity [33]. It shows potent antifungal activity against Candida albicans and Candida glabrata. Similarly, anoplin (GLLKRIKTLL-NH $\mathrm{N}_{2}$ ) isolated from wasp shows potent antimicrobial activity due to its truncation and acylation. Anoplin could be used as promising drug candidates for drug development [34] (Table 2 and figure 2). Anoplin is an amphipathic, $\alpha$-helical bioactive decapeptide isolated from wasp venom. Its shows strong antifungal, activities against Leptosphaeria maculans and protection of Brassica napus plants from disease [36]. Mastoparan analog from wasp venom was also found effective against Candida albicans. It shows low cytotoxicity and non teratogenicity in Danio rerio [35]. Similarly, OdVP2 and OdVP2L peptides were isolated from solitary wasp Orancistrocerus drewseni showed antifungal activity [37] (Figure 2). Polybia-CP is a much potent antifungal peptide. Similar antifungal peptides have been identified in the venom glands of $T$. stigmurus [38]. A peptide Ts-FKLFKKILKVL-NH(2) (BP22), bears Tsortosyl group, shows an efficacy of $56 \%$ disease reduction, than conventional antibiotic. It also shows antifungal activity against both plant-pathogenic and entomopathogenic fungi [30] (Table 2).

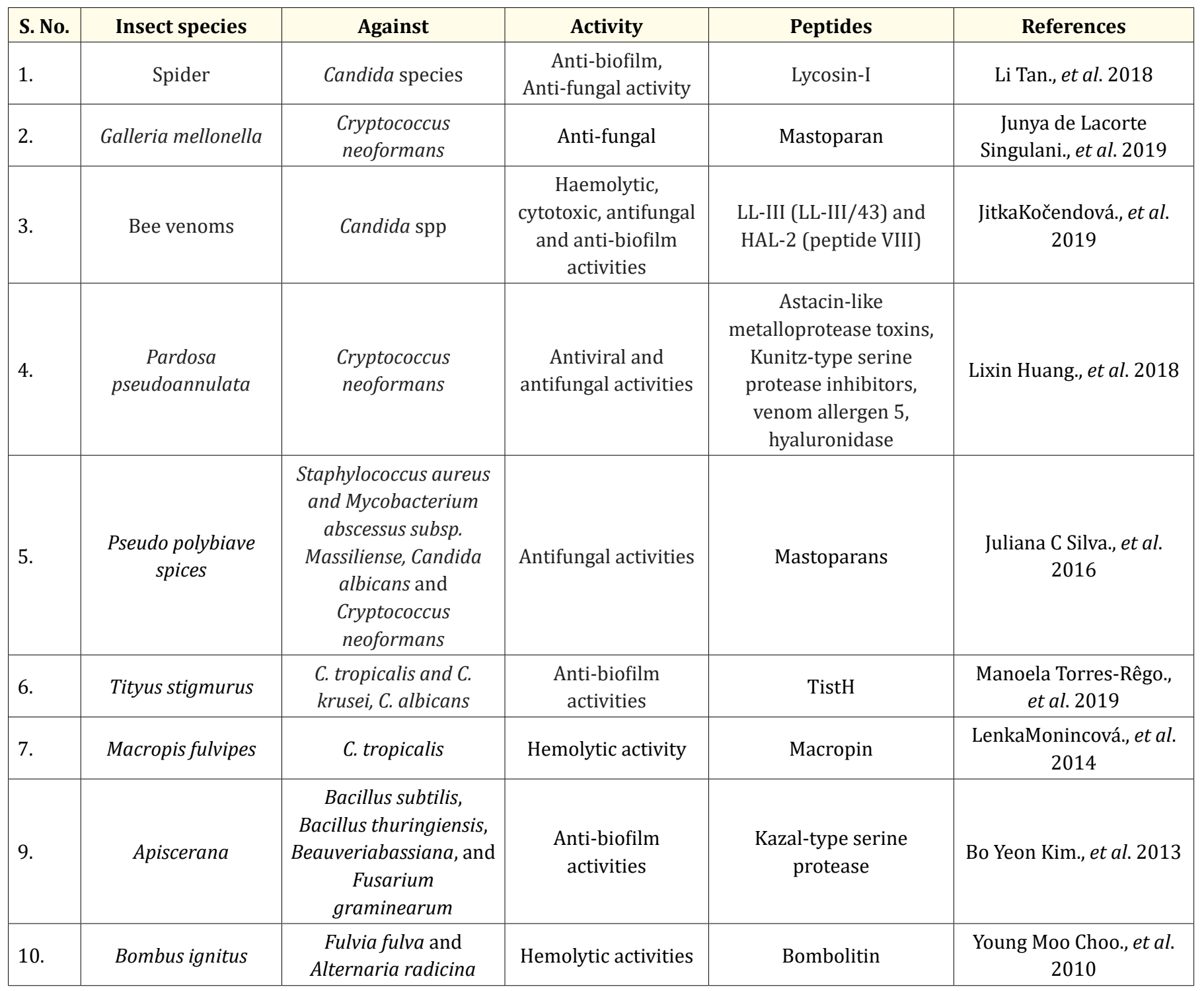




\begin{tabular}{|c|c|c|c|c|c|}
\hline 11. & Lasiodora & $\begin{array}{l}\text { Candida parapsilosis } \\
\text { and Candida albicans }\end{array}$ & $\begin{array}{c}\text { Cytotoxic, and } \\
\text { hemolytic activities }\end{array}$ & $\begin{array}{c}\mathrm{U}_{1} \text {-theraphotoxin-Lp1a } \\
\text { (lasiotoxin-1), } \\
\mathrm{U}_{1} \text {-theraphotoxin-Lp1c } \\
\text { (lasiotoxin-3), } \\
\mathrm{U}_{3} \text {-theraphotoxin- } \\
\text { Lsp1a (LTx5), and } \\
\omega \text {-theraphotoxin-Asp3a, } \\
\text { phospholipase } \mathrm{A}_{2}\left(\mathrm{PLA}_{2} \text { ) }\right. \\
\text { and hyaluronidase }\end{array}$ & $\begin{array}{l}\text { Felipe Roberto Borba } \\
\text { Ferreira., et al. } 2016\end{array}$ \\
\hline 12. & Lachesanatarabaevi & Candida albicans & Cytotoxic, neurotoxic & latarcins (Ltc) & $\begin{array}{l}\text { Peter V Dubovskii., et al. } \\
\qquad 2015\end{array}$ \\
\hline 13. & Polybia paulista & C. tropicalis & $\begin{array}{l}\text { Anti-biofilm } \\
\text { activities }\end{array}$ & polybia-CP & $\begin{array}{l}\text { Kairong Wang., et al. } \\
2015\end{array}$ \\
\hline 16. & $\begin{array}{l}\text { Loxosceles } \\
\text { intermedia }\end{array}$ & Candida parapsilosis & Hemolytic activities & $\begin{array}{l}\text { TAG and DAG } \\
\text { phospholipids }\end{array}$ & A V Bednaski., et al. 2015 \\
\hline 17. & Heterometrus sp. & C. tropicalis & $\begin{array}{l}\text { Anti-biofilm } \\
\text { activities }\end{array}$ & $\kappa$-hefutoxin 1 & Lien Moreels., et al. 2016 \\
\hline
\end{tabular}

Table 2: Anti-fungal activity of animal toxins.

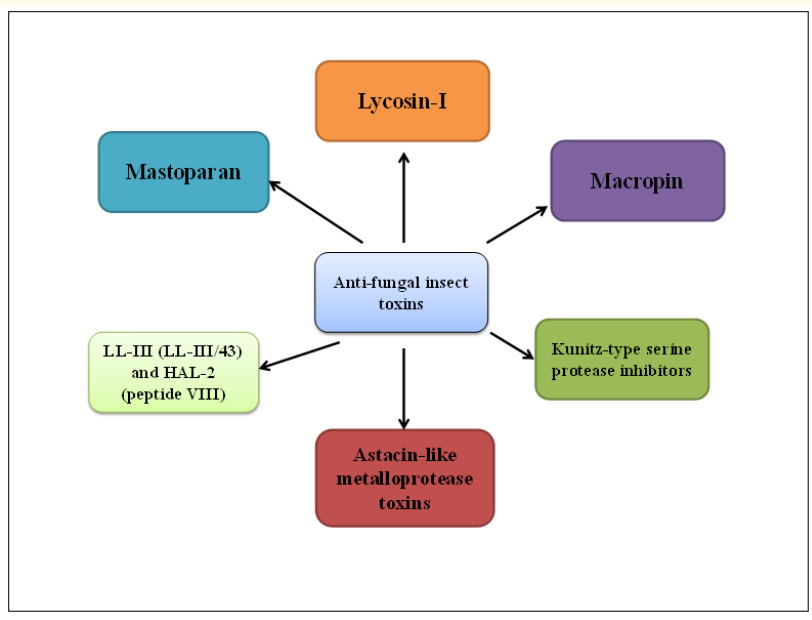

Figure 2: Showing antifungal activity of insect venom toxins.

\section{Anti-trypanosomal}

Mastoparan also shows anti-protozoan activity and inhibits development forms of Trypanosoma cruzi [39]. Venom from the ectoparasitic wasp Nasoniavi tripennis causes cellular injury and involve in the release of intracellular calcium stores via the activation of phospholipase C. It results in oncotic death. Wasp venom stimulates sudden release of calcium from ER compartments, it is distinct from RyRs, $\mathrm{L}$-type $\mathrm{Ca}^{2+}$ channels, and the $\mathrm{Ca}\left({ }^{2+}\right)$-ATPase pump. Calcium is also released from some other intracellular store [40] (Table 3 and figure 3).

\section{Antiviral activity}

Bee venom and its components i.e. melittin (MLT), phospholipase A2 (PLA2), and apamin showed inhibitory effects against important disease causing viruses i.e. Influenza A virus (PR8), Vesicular Stomatitis Virus (VSV), Respiratory Syncytial Virus (RSV), and Herpes Simplex Virus (HSV) in vitro and in vivo [41]. Honey bee peptide melittin found effective against several viruses including coxsackievirus, enterovirus, influenza A viruses, human immunodeficiency virus (HIV), herpes simplex virus (HSV), Junín virus (JV), respiratory syncytial virus (RSV), vesicular stomatitis virus (VSV), and tobacco mosaic virus (TMV) [24,42]. Mastoparan-derived peptide MP7-NH2 inactivates viruses and stimulate cell-mediated 


\begin{tabular}{|l|c|c|c|c|}
\hline S. No. & Insect & Activity & Peptides & References \\
\hline 1. & Ixodes ricinus & Antimalarial, Antiplasmodial & Defensins & Joana Couto., et al. 2018 \\
\hline 2. & Photorhabdus luminescens & $\begin{array}{c}\text { Trypanocidal activity, } \\
\text { parasiticidal activity }\end{array}$ & Bioactive metabolite & $\begin{array}{c}\text { Ana Maria Antonello., et al. } \\
2019\end{array}$ \\
\hline 3. & Xenorhabdus nematophila & $\begin{array}{c}\text { Trypanocidal activity, } \\
\text { parasiticidal activity }\end{array}$ & Bioactive metabolite & $\begin{array}{c}\text { Ana Maria Antonello., } \text { et al. } \\
2019\end{array}$ \\
\hline 4. & Galleria mellonella & Anti-parasitic activity, \\
anti-leishmanial activity & $\begin{array}{c}\text { Moricin-B, Moricin-C4, } \\
\text { Cecropin-D and Anionic } \\
\text { Peptide 2 }\end{array}$ & $\begin{array}{c}\text { Isabel A Patiño-Márquez., } \\
\text { et al. 2018 }\end{array}$ \\
\hline 5. & Achillea fragrantissima & Anti-leishmanial and antitry- \\
panosomal activity & $\begin{array}{c}\text { Sesquiterpene lactones, } \\
\text { flavonoids, chrysosple- } \\
\text { nol-D and chrysosple- } \\
\text { netine, alkamides }\end{array}$ & Joseph Skaf., et al. 2017 \\
\hline
\end{tabular}

Table 3: Anti-protozoal activity of insect venom toxin peptides.

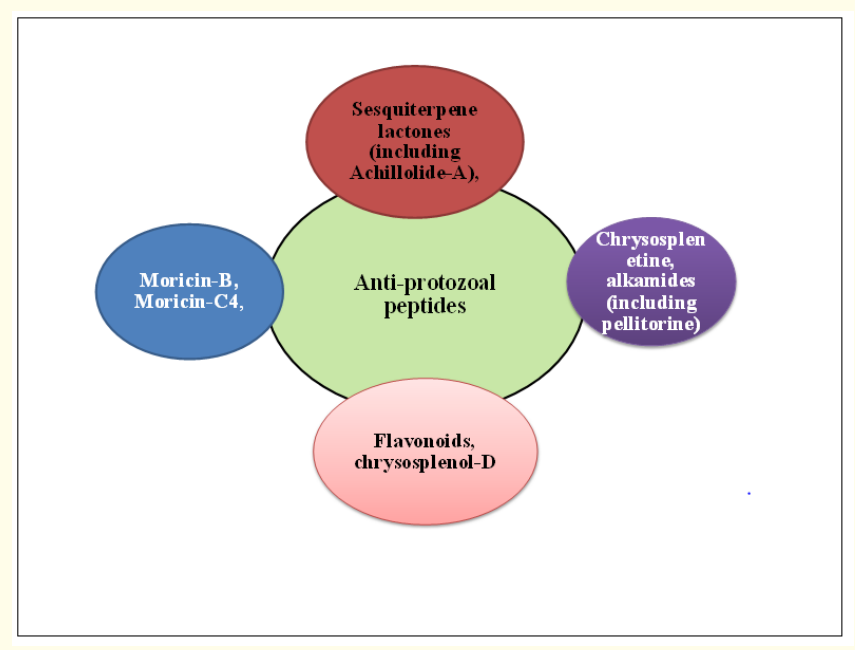

Figure 3: Showing antifungal activity of insect venom toxins.

antiviral defense. MP7-NH2 inactivates mainly enveloped viruses [43] (Table 4 and figure 4). Scorpion-venom-derived mucroporinM1 was found active against RNA viruses (measles viruses, SARSCoV, and H5N1 and HIV-1. Kn2-7 is a scorpion venom peptide that inhibits HIV-1 replication and progression particle [44]. This is a promising candidate development of therapeutic agent against HIV-1 [45]. Cationic peptides showed anti-infection and anti-tumoral activity [46]. T22 [Tyr5,12, Lys7]-polyphemusin II) has been shown to have strong anti-human immunodeficiency virus (HIV) activity. T22 inhibits the T cell line-tropic (T-tropic) HIV-1 infection through its specific binding to a chemokine receptor CXCR4, which serves as a coreceptor for the entry of T-tropic HIV-1 strains. Bee venom toxins show biological activities mainly antiviral [47] (Table 4 and figure 4).

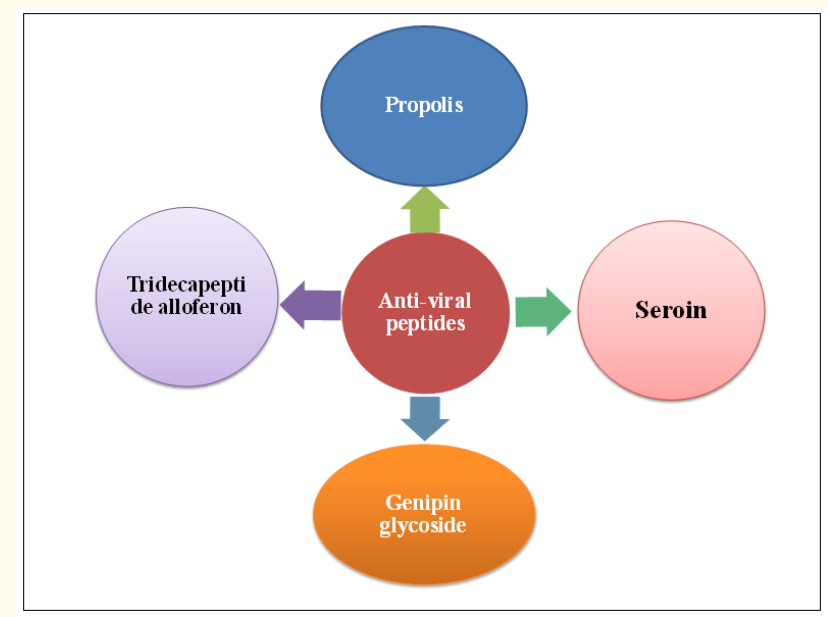

Figure 4: Showing antiviral activity of insect venom toxins.

Tick saliva also contains defensins against microbes mainly viruses. It plays a major role in the innate immunity of ticks [48]. T22 [Tyr5,12, Lys7]-polyphemusin II) inhibits human immuno- 


\begin{tabular}{|c|c|c|c|c|}
\hline S. No. & Insect & Biological effect & Peptides & References \\
\hline 1. & Apis mellifera & Cytotoxic and anti-herpetic effect & Propolis & K Labska., et al. 2018 \\
\hline 2. & Bombyx mori & Cytotoxicity & Seroin & C P Singh., et al. 2014 \\
\hline 4. & Drosophila & Antiviral immunity, & & Alfred W Bronkhorst., et al. 2019 \\
\hline 5. & & $\begin{array}{l}\text { Larvacidal activities, insecticidal } \\
\text { activity }\end{array}$ & Genipin glycoside & Qing Xia., et al. 2018 \\
\hline 6. & Apis mellifera & $\begin{array}{c}\text { Anti oxidant activity and irritation } \\
\text { property }\end{array}$ & Melittin & Alexander J McMenamin., et al. 2018 \\
\hline 7. & Apis cerana & Antiviral immunity & Cecropin A1 & Alexander J McMenamin., et al. 2018 \\
\hline 8. & Bombus sp & Antibacterial, antiviral & Prodigiosin & Alexander J McMenamin., et al. 2018 \\
\hline 9. & Apis mellifera & $\begin{array}{c}\text { Antibacterial, antiviral, anti } \\
\text {-inflammatory, antiallergic, and } \\
\text { vasodilatory actions }\end{array}$ & Propolis, flavonoids & M Viuda-Martos., et al. 2018 \\
\hline 11. & $\begin{array}{l}\text { Haemaphysalis } \\
\text { longicornis }\end{array}$ & Virucidal activity & $\begin{array}{l}\text { Defensin-like peptide, } \\
\text { HEdefensin }\end{array}$ & Melbourne Rio Talactac., et al. 2017 \\
\hline 12. & Bombyx mori & Antiviral activity against BmNPV & Prodigiosin & Wei Zhou., et al. 2016 \\
\hline 13. & Lonomia obliqua & Antiviral activity & Defensin-like peptide & A C V Carmo., et al. 2012 \\
\hline 14. & Bombyx mori L & Antiviral, antifungal & $\begin{array}{l}\text { Red fluorescent protein } \\
\text { (RFP }\end{array}$ & G K S Manjunatha., et al. 2018 \\
\hline 15. & Musca domestica & In vitro anti-influenza activity & & FurongWang., et al. 2013 \\
\hline
\end{tabular}

Table 4: Anti-viral activity of insect venom toxin peptides.

deficiency virus (HIV) activity. T22 inhibits replication of HIV-1 through its specific binding to a chemokine receptor CXCR4, which serves as a co-receptor for the entry of T-tropic HIV-1 strains. Prodigiosin showed antiviral activity Bombyx mori nucleopolyhedrovirus (BmNPV)-infected cells in vitro, with specific modes of action [49]. The hemolymph of Lonomia oblique caterpillars contain an antiviral protein. This antiviral protein needs baculovirus/Sf9 cell system for expression [50].

The midgut mucosa and serosa and other membrane structures acts as natural barrier of pathogens and assist in making innate immune defense. Red fluorescent protein (RFP) is purified from the digestive juice of $B$. mori larvae. It shows antifungal and antibacterial properties. N-terminal sequence of RFP analysis having chbp gene and it belongs to lipocalin gene family found involve in anti-pathogenic activities. RFP from the midgut of the silkworm larvae showed antiviral activity against the BmNPV. It also shows antibacterial activity against, Klebsiella pneumonia, Bacillus subtilis and, Phytophthora meadii [51]. PEF (protein-enriched fraction) isolated from the larvae of the housefly showed strong antiviral activity against influenza virus. It stops virus entry into the cells. PEF 
has a great potential as a resource of healthy products [52] (Table 4). BmSP-2 is an insect digestive enzyme that acts as an antiviral factor against BmNPV at the initial site of viral infection [52]. Rbv protein is isolated from hemolymph of Lonomia oblique; it shows potent antiviral activity against measles, influenza and polio viruses [53]. This protein function as a constitutive agent to make the innate immune defense. These effects may be mediated by changes in the GTP pools of treated cells [54]. Seroins are proteins isolated from Bombyx mori show strong anti-viral activity. Seroins could be used as potent candidates for development of transgene-based disease resistant silkworm strains [55]. Melittin and phospholipase A2 (PLA2) are major components of bee venom. Bees and wasps venom also contain anti-cancer peptides (ACPs), most of them are small cationic and hydrophobic peptides which show antioxidant, antimicrobial, neuroprotective or antitumor effects [56] (Table 4).

\section{Anti-cancer peptides}

Anti-cancer peptides (ACPs) are small cationic and hydrophobic peptides. Anticancer peptides much ably kill cancer cells by causing irreparable membrane damage and cell lysis, or by inducing apoptosis. Mastoparan induce mast cell granulation through exocytosis (Table 5). It facilitates mitochondrial permeability through a transition opening of a pore [57].

\section{Miscellaneous}

Animal venoms possess hundreds of molecules, mostly peptides which display diverse biological activities mainly pharmaco-

\begin{tabular}{|c|c|c|c|c|}
\hline Species & Peptides & Biological effects & Activity & References \\
\hline $\begin{array}{l}\text { Solitory wasp and hunting } \\
\text { Wasps }\end{array}$ & $\begin{array}{c}\text { Pompilidotoxin and } \\
\text { dendrotoxin-like peptide), } \\
\text { insulin-like peptide binding } \\
\text { protein) }\end{array}$ & $\begin{array}{l}\text { Active against bacteria } \\
\text { and fungi }\end{array}$ & $\begin{array}{c}\text { Antimicrobial } \\
\text { Peptide } \\
\text { Neurotoxic peptides }\end{array}$ & $\begin{array}{c}\text { Si Hyeock } \\
\text { Lee., et al., } 2016\end{array}$ \\
\hline $\begin{array}{l}\text { Neotropical Social Wasp } \\
\text { Polybia dimorpha }\end{array}$ & Polydim-I & $\begin{array}{l}\text { Cytotoxic effects, cell } \\
\text { wall disruption }\end{array}$ & Anti-mycobacterial & $\begin{array}{l}\text { Rogerio Coutinho } \\
\text { das Neves., et al. } \\
2016\end{array}$ \\
\hline Polybia paulista & Polybia-CP & $\begin{array}{c}\text { Membrane-Active Action } \\
\text { Mode }\end{array}$ & $\begin{array}{l}\text { Antimicrobial } \\
\text { Peptide }\end{array}$ & $\begin{array}{l}\text { Kairong Wang., et al. } \\
2012\end{array}$ \\
\hline $\begin{array}{l}\text { Social wasp Vespula } \\
\text { vulgaris }\end{array}$ & Mastoparan V1 (MP-V1), & $\begin{array}{c}\text { Anti-Salmonella Activity } \\
\text { Modulation }\end{array}$ & $\begin{array}{l}\text { antimicrobial } \\
\text { peptide }\end{array}$ & $\begin{array}{c}\text { Yeon Jo Ha., et al. } \\
2017\end{array}$ \\
\hline $\begin{array}{l}\text { Solitary eumenine } \\
\text { wasp Anterhynchium } \\
\text { flavo marginatum micado }\end{array}$ & Eumeninemastoparan-AF & $\begin{array}{l}\text { histamine-releasing } \\
\text { factor }\end{array}$ & $\begin{array}{l}\text { Antimicrobial, and } \\
\text { hemolytic }\end{array}$ & $\begin{array}{l}\text { Marcia Perez dos } \\
\text { Santos Cabrera., } \\
\quad \text { et al. } 2019\end{array}$ \\
\hline $\begin{array}{l}\text { Solitary eumenine } \\
\text { wasp Eumenesmicado }\end{array}$ & $\begin{array}{c}\text { Eumenine } \\
\text { mastoparan-EM1 (and } \\
\text { eumenine } \\
\text { mastoparan- } \\
\text { EM2,mastoparan peptides, } \\
\text { linear cationic } \alpha \text {-helical } \\
\text { peptides }\end{array}$ & $\begin{array}{c}\text { Moderate degranulation } \\
\text { activity, leishmanicidal } \\
\text { activity }\end{array}$ & Antibacterial activity & $\begin{array}{l}\text { Katsuhiro Konno., } \\
\text { et al. } 2019\end{array}$ \\
\hline $\begin{array}{l}\text { Parasitoid wasps Leptopilin } \\
\text { aboulardi }\end{array}$ & Edin & Cellular cytotoxicity & $\begin{array}{l}\text { Mast cell (MC) } \\
\text { degranulation }\end{array}$ & $\begin{array}{c}\text { Leena } \\
\text {-MaijaVanha-aho., et } \\
\text { al. } 2015\end{array}$ \\
\hline $\begin{array}{l}\text { Endoparasitoid wasp, } \\
\text { Microplitis mediator }\end{array}$ & $\begin{array}{l}\text { VRF1, a metalloprotease } \\
\text { homolog venom protein }\end{array}$ & $\begin{array}{l}\text { Toll signaling pathway in } \\
\text { the host hemocytes }\end{array}$ & Anti viral & Zhe Lin., et al. 2018 \\
\hline
\end{tabular}




\begin{tabular}{|c|c|c|c|c|}
\hline $\begin{array}{l}\text { The wild bee Panurgus } \\
\text { calcaratus. }\end{array}$ & Panurgines & $\begin{array}{l}\text { Hemolytic activity } \\
\text { against human } \\
\text { erythrocytes. }\end{array}$ & $\begin{array}{l}\text { Antifungal } \\
\text { therapeutic, } \\
\text { antimicrobial } \\
\text { peptides }\end{array}$ & Čujová S., et al. 45 \\
\hline $\begin{array}{l}\text { Lasioglossum laticeps (wild } \\
\text { bee) }\end{array}$ & Lasiocepsin & $\begin{array}{c}\text { Membrane } \\
\text {-permeabilising activity }\end{array}$ & $\begin{array}{l}\text { Antibacterial and } \\
\text { antifungal activity }\end{array}$ & Bednaski AV., et al. \\
\hline Pseudo polybiavespiceps & Mastoparans, Polybia-MPII & $\begin{array}{c}\text { Potent action against } \\
\text { S. aureus, apoptosis and } \\
\text { inflammation }\end{array}$ & antimicrobial drugs, & $\begin{array}{l}\text { Juliana C Silva., et al. } \\
\qquad 2017\end{array}$ \\
\hline Social wasp Polybia paulista & Polybia-CP & $\begin{array}{c}\text { Membrane-active action } \\
\text { mode }\end{array}$ & Antifungal activity & $\begin{array}{l}\text { Kairong Wang., et al. } \\
2014 .\end{array}$ \\
\hline $\begin{array}{l}\text { Solitary wasp } \\
\text { Orancistrocerusdrewseni }\end{array}$ & OdVP1, OdVP2 and OdVP3 & $\begin{array}{c}\text { Moderate degranulation } \\
\text { activity, leishmanicidal } \\
\text { activity }\end{array}$ & $\begin{array}{l}\text { Strong antifungal } \\
\text { activities }\end{array}$ & $\begin{array}{l}\text { JiHyeongBaek., et al. } \\
2010\end{array}$ \\
\hline $\begin{array}{l}\text { Polybia paulista wasp } \\
\text { venom }\end{array}$ & Mastoparan & Parasitic infection & Anti trypanosomal & $\begin{array}{c}\text { Juliana Freire Chagas } \\
\text { Vinhote., et al. } 2017\end{array}$ \\
\hline $\begin{array}{l}\text { Ectoparasitic wasp Nasonia } \\
\text { vitripennis }\end{array}$ & Metalloprotease & $\begin{array}{l}\text { The activation of } \\
\text { phospholipase } \mathrm{C} \text {, and } \\
\text { culminates in oncotic } \\
\text { death }\end{array}$ & Anti trypanosomal & $\begin{array}{l}\text { David B Rivers" et al. } \\
2005 .\end{array}$ \\
\hline
\end{tabular}

Table 5: Wasp venom peptides.

logical and therapeutic [58]. Polistine wasps use venom stinging as an effective weapon against predators. It contains neurotoxin which are haemolytic, and algogenic in nature [59]. Honey, propolis, and royal jelly, contain phenolic compounds mainly flavonoids [59]. These show anti-bacterial, antiviral, anti-inflammatory, antiallergic and vasodilatory activities [60]. Flavonoids inhibit important physiological activities such as lipid peroxidation, platelet aggregation, capillary permeability and fragility. It also inhibits cyclo-oxygenase and lipoxygenase enzyme activity [60]. Honeybees prepare propolis a resinous substance that is used in traditional medicine against Varicella zoster virus [61]. It shows antimicrobial, anti-inflammatory, and immune modulatory effects.

Royal jelly shows antibacterial, anti-inflammatory, anti-hypercholesterolemic, vasodilative and hypotensive activities. Honey bee venom components prepare antiviral defense by using RNA interference (RNAi), endocytosis, melanization, encapsulation and autophagy. It also operates through conserved immune pathways including Jak/STAT (Janus kinase/signal transducer and activator of transcription), JNK (c-Jun N-terminal kinase), MAPK (mitogenactivated protein kinases) and the NF-kB mediated Toll and im- mune deficiency pathways [62] (Table 5).

Mellitin, a major component isolated from bee venom, Apis mellifera, shows antimicrobial activity against gram positive bacteria. Insects also synthesize variety of immune-induced molecules which show antibacterial and antifungal property [63]. Cationic peptides are also known as alloferons. These were isolated from blow fly Calliphora vicina (Diptera) (Table 5).Crude venom of wasp Nasonia vitripennis shows lethal effects. Wasps, bees and hornet venom shows hemolytic activity to Sarcophaga peregrina (NIH SaPe4), Drosophila melanogaster (CRL 1963), Trichoplusia ni (TN368 and BTI-TN-5B1-4), Spodoptera frugiperda (SF-21AE), and Lymantria dispar (IPL-Ldfbc1) cells [64]. Moreover, peptides and proteins such as pilosulin-like peptides, phospholipase $\mathrm{A}_{2} \mathrm{~s}$, hyaluronidase, venom dipeptidyl peptidases, conotoxin-like peptide, and icarapin-like peptide antimicrobial, hemolytic, and histaminereleasing activities. Pilosulin-like peptides 1-6 were chemically synthesized and some of them displayed antimicrobial, hemolytic, and histamine-releasing activities [65] (Table 5). These could be used as important tools for the development of new therapeutic drugs. 


\section{Mode of action of peptides}

Insect toxins comprise a diverse array of chemicals ranging from small molecules, polyamines and peptide toxins. Many of these venom components target nervous system and neuromuscular ion channels and so rapidly affect the behavior of animals to which the toxin is applied or injected. Melittin, acts on the membrane surface of nerve cells while mastoparan shows strong cytolytic effects. Philanthotoxins found in digger wasps specially target ligand-gated ion channels. These act on nervous system and at neuromuscular junctions. Apamin isolated from bee venom acts upon calcium-activated potassium channels, and influence release of neuropeptides. The crude honey bee (Apis mellifera) shows effects on skeletal, smooth as well as cardiac muscles. Crude venom also causes neurotoxicity and inhibits autonomic as well as neuromuscular system. Honey bee venom, shows induction of apoptosis in malignant cells. It shows inhibitory, anti-invasive and cytotoxic effect on several types of cancer lines. Bee venom components melittin shows showed anti-proliferative and anti-metastatic properties and apoptosis in malignant glioma cells.

\section{Mastoparan}

Mastoparan destroys bilayer membranes and come across through pores. Both low and high concentrations of mastoparan affect membrane permeabilization through transition pore. Gprotein participates in regulation of the permeability through transition pore [66]. Mastoparan severely affects mitochondrial permeability through bi-functional mechanism. Polybia-MPII induces myonecrosis and apoptosis, by involving caspases signaling activity. It results in mitochondrial damage, and cytokine activation [66]. This peptide binds to membrane lipids due to electrostatic attraction. It partially accumulates, neutralizes the opposite charges and induces pore formation in biological membrane. Mastoparan as an inducer of mast cell granules exocytosis has been also related to many essential mechanisms of cell function [57].

\section{Cationic polydim-I}

This peptide binds to membrane lipids due to electrostatic attraction. It partially accumulates, neutralizes the opposite charges and induces pore formation in biological membrane. It could be used against multi-drug resistant pathogens mainly bacteria in the hospital environment [67].

\section{Brefeldin A}

It interferes in Golgi apparatus endoplasmic reticulum operated functions. Mastoparan activates heterotrimeric G proteins, promotes binding of beta-COP to Golgi membranes in vitro. It antagonizes the effect of brefeldin A on beta-COP in perforated cells as well as in isolated Golgi membranes [68].

\section{Sifuvirtide}

Sifuvirtide stops HIV-1 fusion. It interacts with lipid structures during fusion after its delivery [69].

\section{Prodigiosin}

Prodigiosin selectively target virus-infected cells, inhibit viral gene transcription, and virus-mediated membrane fusion. Prodigiosin found cell cytoplasm where it interact with cytoplasm factors and inhibit virus replication [70].

\section{Conclusion}

Insect venom toxin peptides showed diverse biological activity i.e. vasodilatory, anti-septic and cytotoxic hypotensive, antioxidant activity, anti-allergic, anti-hypercholesterolemic, anti-rheumatic and analgesic, anticancer effects. Insect venom toxins also possess remarkable antibacterial, antifungal antiviral, and insecticidal activity. Antimicrobial peptides isolated from venoms of different insects could be used as useful resources for new anti-infective agents. Due to diverse biological activity of insect venom toxins and its fast action of venom toxins make them important for the pharmaceutical industry. No doubt antimicrobial peptides isolated from venoms of different hymenopteran insects could be used for designing anti-infective therapeutic agents. These peptides could be used as templates for designing appropriate and more potent structures by using drug design and drug delivery systems. For pharmacological use of venom toxins, component specific effects are to be explored in various animal models. It is only possible after coordinating efforts being done in the field of biochemistry, pharmacology and immunology. This wider knowledge on insect toxins will help in production of new highly potent drug molecules to fight against various disease pathogens and will open new ways and innovations in the field of therapeutic and pharmaceutical research.

\section{Acknowledgements}

Authors are thankful to H.O.D., Department of Zoology for research facilities. 


\section{Conflict of Interest}

The authors declare no competing financial interests.

\section{Bibliography}

1. Lee SH., et al. "Differential Properties of Venom Peptides and Proteins in Solitary vs. Social Hunting Wasps”. Toxins (Basel) 8 (2016): 8020032.

2. Monincová L., et al. "Structure-activity study of macropin, a novel antimicrobial peptide from the venom of solitary bee Macropisfulvipes (Hymenoptera: Melittidae)". Journal of Peptide Science 20 (2014): 375-84.

3. Kim BY., et al. "Antimicrobial activity of a honeybee (Apiscerana) venom Kazal-type serine protease inhibitor". Toxicon (2013): 110-117.

4. Čujová S., et al. "Panurgines, novel antimicrobial peptides from the venom of communal bee Panurguscalcaratus (Hymenoptera: Andrenidae)". Amino Acids 45 (2013): 143-157.

5. Câmara GA., et al. "A Multiomics Approach Unravels New Toxins With Possible InSilico Antimicrobial, Antiviral, and Antitumoral Activities in the Venom of Acanthoscurriarondoniae". Frontiers in Pharmacology 11 (2020): 1075.

6. Silva JC., et al. "Evaluation of the antimicrobial activity of the mastoparanPolybia-MPII isolated from venom of the social wasp Pseudo polybiavespicepstestacea (Vespidae, Hymenoptera)". International Journal of Antimicrobial Agents 49 (2017): 167-175.

7. Das Neves RC., et al. "Antimycobacterial Activity of a New Peptide Polydim-I Isolated from Neotropical Social Wasp Polybiadimorpha". PLoS One 11 (2016): e0149729.

8. Ha YJ., et al. "Anti-Salmonella Activity Modulation of Mastoparan V1-A Wasp Venom Toxin-Using Protease Inhibitors, and Its Efficient Production via an Escherichia coli Secretion System". Toxins (Basel) 9 (2017): 321.

9. Rangel M., et al. "Polydim-I antimicrobial activity against MDR bacteria and its model membrane interaction". PLoS One 12 (2017) :e0178785

10. Dos Santos Cabrera MP., et al. "Chemical and Biological Characteristics of Antimicrobial $\alpha$-Helical Peptides Found in Solitary
Wasp Venoms and Their Interactions with Model Membranes". Toxins (Basel) 11 (2019): 559.

11. Konno K., et al. "New mastoparan peptides in the venom of the Solitary Eumenine Wasp Eumenesmicado". Toxins (Basel) 10 (2019): 155.

12. Kim BY., et al. "Antimicrobial activity of a honeybee (Apiscerana) venom Kazal-type serine protease inhibitor". Toxicon (2013): 110-117.

13. Cesa-Luna C., et al. "Structural characterization of scorpion peptides and their bactericidal activity against clinical isolates of multidrug-resistant bacteria". PLoS One 11 (2019): e0222438.

14. Zhang S., et al. "Loop Replacement Enhances the Ancestral Antibacterial Function of a Bifunctional Scorpion Toxin". Toxins (Basel) 4 (2018): 227.

15. Dubovskii PV., et al. "Latarcins: versatile spider venom peptides". Cellular and Molecular Life Sciences 72 (2015): 45014522.

16. Monincová L., et al. "Structural basis for antimicrobial activity of lasiocepsi”. Chembiochem 15 (2014): 301-318.

17. Ha YJ., et al. "Anti-Salmonella Activity Modulation of Mastoparan V1-A Wasp Venom Toxin-Using Protease Inhibitors, and Its Efficient Production via an Escherichia coli Secretion System". Toxins (Basel) 9 (2017): 321.

18. Rangel M., et al. "Polydim-I antimicrobial activity against MDR bacteria and its model membrane interaction". PLoS One 12 (2017): e0178785.

19. Konno K., et al. "New Mastoparan Peptides in the Venom of the Solitary Eumenine Wasp Eumenes micado". Toxins (Basel) 11 (2019): 155.

20. Madden AA., et al. "The emerging contribution of social wasps to grape rots disease ecology". Peer Journal 5 (2017): e3223.

21. Lin Z., et al. "A Metalloprotease Homolog Venom Protein From a Parasitoid Wasp Suppresses the Toll Pathway in Host Hemocytes". Frontiers in Immunology 9 (2018): 2301.

22. Vanha-Aho LM., et al. "Edin Expression in the Fat Body Is Required in the Defense Against Parasitic Wasps in Drosophila melanogaster". PLoS Pathogen 11 (2015): e1004895. 
23. Linial M., et al. “Overlooked Short Toxin-Like Proteins: A Shortcut to Drug Design". Toxins (Basel) 9 (2017): 350.

24. Wehbe R., et al. "Bee Venom: Overview of Main Compounds and Bioactivities for Therapeutic Interests". Molecules 24 (2019): 24162997.

25. Fischer ML., et al. "Context-dependent venom deployment and protein composition in two assassin bugs". Ecology and Evolution 10 (2020): 9932-9947.

26. Wang K., et al. "Membrane-active action mode of polybia$\mathrm{CP}$, a novel antimicrobial peptide isolated from the venom of Polybiapaulista". Antimicrobial Agents and Chemotherapy 56 (2012): 3318-3323.

27. Das Neves RC., et al. "Antimicrobial and Antibiofilm Effects of Peptides from Venom of Social Wasp and Scorpion on Multidrug-Resistant Acinetobacterbaumannii". Toxins (Basel) 11 (2019): 216.

28. Munk JK., et al. "Synthetic analogs of anoplin show improved antimicrobial activities". Journal of Peptide Science 19 (2013): 669-675.

29. Moerman L., et al. "Antibacterial and antifungal properties of alpha-helical, cationic peptides in the venom of scorpions from southern Africa". FEBS Journal 269 (2002): 4799-4810.

30. Badosa E, et al . "Sporicidal activity of synthetic antifungal undecapeptides and control of Penicillium rot of apples". Applied and Environmental Microbiology 75 (2009): 5563-5569.

31. Moreels L., et al. "Expanding the pharmacological profile of $\kappa$-hefutoxin 1 and analogues: A focus on the inhibitory effect on the oncogenic channel Kv10.1". Peptides 98 (2017): 43-50.

32. Marcos CM., et al. "Down-regulation of TUFM impairs host cell interaction and virulence by Paracoccidioidesbrasiliensis". Scientific Report 9 (2019): 17206.

33. Wang K., et al. "Dual antifungal properties of cationic antimicrobial peptides polybia-MPI: membrane integrity disruption and inhibition of biofilm formation". Peptides 56 (2014): 22-29.

34. Jindřichová B., et al. "Novel properties of antimicrobial peptide anoplin". Biochemical and Biophysical Research Communications 444.4 (2014): 520-524.
35. Salas RL., et al. "Effects of truncation of the peptide chain on the secondary structure and bioactivities of palmitoylatedanoplin". Peptides 104 (2018): 7-14.

36. GaleaneMC,et al. "Study of mastoparan analog peptides against Candida albicans and safety in zebrafish embryos (Daniorerio)". Future Microbiology 14 (2019): 1087-1097.

37. Baek JH., et al. "Isolation and molecular cloning of venom peptides from Orancistrocerusdrewseni (Hymenoptera: Eumenidae)". Toxicon 55 (2010): 711-718.

38. Torres-Rêgo M., et al. "Biodegradable cross-linked chitosan nanoparticles improve anti-Candida and anti-biofilm activity of TistH, a peptide identified in the venom gland of the Tityus stigmurus scorpion". Materials Science and Engineering C 103 (2019): 109830.

39. Vinhote JFC., et al. "Trypanocidal activity of mastoparan from Polybiapaulista wasp venom by interaction with TcGAPDH". Toxicon 137 (2017): 168-172.

40. Rivers DB., et al. "Localization of intracellular calcium release in cells injured by venom from the ectoparasitoidNasoniavitripennis (Walker) (Hymenoptera: Pteromalidae) and dependence of calcium mobilization on G-protein activation". Journal of Insect Physiology 51 (2005): 149-160.

41. Uddin MB., et al. "Inhibitory effects of bee venom and its components against viruses invitro and in vivo". Journal of Microbiology 54 (2016): 853-866.

42. Lee WR., et al. "The protective effects of melittin on Propionibacterium acnes-induced inflammatory responses in vitro and in vivo". Journal of Investigative Dermatology 134 (2014): 1922-1930.

43. Sarhan M., et al. "Potent virucidal activity of honeybee Apismellifera" venom against Hepatitis C Virus". Toxicon 188 (2020): 55-64.

44. Sample CJ., et al. "A mastoparan-derived peptide has broadspectrum antiviral activity against enveloped viruses". Peptides 48 (2013): 96-105.

45. Chen Y., et al. "Anti-HIV-1 activity of a new scorpion venom peptide derivative Kn2-7". PLoS One 7 (2012): e34947. 
46. Couto J., et al. "Anti-plasmodial activity of tick defensins in a mouse model of malaria". Ticks and Tick-borne Diseases 9 (2018): 844-849.

47. Tamamura H., et al. "Analysis of the interaction of an anti-HIV peptide, T22 ([Tyr5, 12, Lys7]-polyphemusin II), with gp120 and CD4 by surface plasmon resonance". Biochimica et Biophysica Acta (BBA) - Protein Structure and Molecular Enzymology 1298 (1996): 37-44.

48. Zhou W., et al. "Antiviral activity and specific modes of action of bacterial prodigiosin against Bombyxmorinucleopolyhedrovirus in vitro". Applied Microbiology and Biotechnology 100 (2016): 3979-3988.

49. Carmo AC, et al. "Expression of an antiviral protein from Lonomia obliquehemolymph in baculovirus/insect cell system". Antiviral Research 94 (2012): 126-130.

50. Manjunatha GKS., et al. "Identification of In-Vitro Red Fluorescent Protein with Antipathogenic Activity from the Midgut of the Silkworm (Bombyx Mori L.)". Protein and Peptide Letters 25 (2018): 302-313.

51. Wang F., et al. "In vitro anti-influenza activity of a protein-enriched fraction from larvae of the housefly (Muscadomestica)". Pharmaceutical Biology 51 (2013): 405-410.

52. Nakazawa H., et al. "Antiviral activity of a serine protease from the digestive juice of Bombyxmori larvae against nucleopolyhedrovirus". Virology 321 (2004): 154-162.

53. Greco KN., et al. "Antiviral activity of the hemolymph of Lonomiaobliqua (Lepidoptera: Saturniidae)". Antiviral Research 84 (2009): 84-90.

54. Liao HJ., et al. "Reversal of the antiviral activity of ribavirin against Sindbis virus in Ae. albopictus mosquito cells". Antiviral Research 22 (1993): 285-294.

55. Singh CP., et al. "Characterization of antiviral and antibacterial activity of Bombyxmoriseroin proteins". Cell Microbiology 16 (2014): 1354-1365.

56. Carpena M., et al. "Bee Venom: An Updating Review of Its Bioactive Molecules and Its Health Applications". Nutrients 12 (2020): 3360 .
57. Hilchie AL., et al. "Mastoparan is a membranolytic anti-cancer peptide that works synergistically with gemcitabine in a mouse model of mammary carcinoma". Biochimica et Biophysica Acta 1858 (2016): 3195-3204.

58. da Mata ÉC., et al. "Antiviral activity of animal venom peptides and related compounds". Journal of Venomous Animals and Toxins including Tropical Diseases 23 (2017): 3.

59. Ogundeyi S.B. et al. "Effect of Polistesfuscatus (Hymenoptera: wasp)" (2017).

60. Viuda-Martos M., et al. "Functional properties of honey, propolis, and royal jelly”. Journal of Food Science 73 (2008): 117-124.

61. Labská K., et al. "Antiviral activity of propolis special extracts GH 2002 against Varicella zoster virus in vitro". Pharmazie 73 (2018): 733-736.

62. McMenamin AJ., et al. "Honey Bee and Bumble Bee Antiviral Defense”. Viruses 10 (2018): 395.

63. Zolfagharian H., et al. "Bee Venom (Apismellifera) an Effective Potential Alternative to Gentamicin for Specific Bacteria Strains: Bee Venom an Effective Potential for Bacteria”. Journal of Pharmacopuncture 19 (2016): 225-230.

64. Rivers DB., et al. "In vitro analysis of venom from the wasp Nasoniavitripennis: susceptibility of different cell lines and venom-induced changes in plasma membrane permeability". In Vitro Cellular and Developmental Biology - Animal 35 (1999): 102-110.

65. Tani N., et al. "Mass Spectrometry Analysis and Biological Characterization of the Predatory Ant Odontomachusmonticola Venom and Venom Sac Components". Toxins (Basel) 11 (2019): 50 .

66. Pfeiffer DR., et al. "The peptide mastoparan is a potent facilitator of the mitochondrial permeability transition". Journal of Biological Chemistry 270 (1995): 4923-4932.

67. Rangel M., et al. "Polydim-I antimicrobial activity against MDR bacteria and its model membrane interaction". PLoS One 12 (2017): e0178785.

68. Ktistakis NT., et al. "Action of brefeldin A blocked by activation of a pertussis-toxin-sensitive G protein". Nature 356 (1992): 
344-346.

69. Avram S., et al. "Evaluation of the Therapeutic Properties of Mastoparan- and Sifuvirtide- Derivative Antimicrobial Peptides Using Chemical Structure-Function Relationship - in vivo and in silico Approaches". Current Drug Delivery 13 (2016):

202-210.

70. Gerber NN. "Prodigiosin-like pigments". Critical Reviews in Microbiology 3.4 (1975): 469-485.

\section{Assets from publication with us}

- Prompt Acknowledgement after receiving the article

- Thorough Double blinded peer review

- Rapid Publication

- Issue of Publication Certificate

- High visibility of your Published work

Website: www.actascientific.com/

Submit Article: www.actascientific.com/submission.php

Email us: editor@actascientific.com

Contact us: +919182824667 